OPEN ACCESS

Edited by: Nobuhiro Suzuki,

Okayama University, Japan

Reviewed by:

Daohong Jiang,

Huazhong Agricultural University,

China

Hiromitsu Moriyama

Tokyo University of Agriculture and

Technology, Japan

Michael Norman Pearson,

University of Auckland, New Zealand

*Correspondence:

loly Kotta-Loizou

i.kotta-loizou13@imperial.ac.uk

Specialty section:

This article was submitted to

Virology,

a section of the journal

Frontiers in Microbiology

Received: 17 June 2017

Accepted: 23 August 2017

Published: 06 September 2017

Citation:

Kotta-Loizou I and Coutts RHA (2017)

Mycoviruses in Aspergilli: A

Comprehensive Review.

Front. Microbiol. 8:1699.

doi: 10.3389/fmicb.2017.01699

\section{Mycoviruses in Aspergilli: A Comprehensive Review}

\author{
loly Kotta-Loizou ${ }^{1 *}$ and Robert H. A. Coutts ${ }^{2}$ \\ ${ }^{1}$ Department of Life Sciences, Imperial College London, London, United Kingdom, ${ }^{2}$ Department of Biological and \\ Environmental Sciences, University of Hertfordshire, Hatfield, United Kingdom
}

Fungi, similar to all species, are susceptible to viral infection. Aspergillus is arguably the most well studied fungal genus because of its medical, ecological and economical significance. Mycoviruses were initially detected in Aspergillus species almost 50 years ago and the field continues to be active today with ground-breaking discoveries. The aim of the present review is to cover the scientific progress in all aspects of mycovirology as exemplified by Aspergillus-focused research. Initially an overview of the population studies illustrating the presence of mycoviruses in numerous important Aspergillus species, such as A. niger, A. flavus, and A. fumigatus with be presented. Moreover the intricacies of mycovirus transmission, both inter- and intra-species, will be discussed together with the methodologies used to investigate viral dispersion in a laboratory setting. Subsequently, the genomic features of all molecularly characterized mycoviruses to date will be analyzed in depth. These include members of established viral families, such as Partitiviridae, Chrysoviridae and Totiviridae, but also more recent, novel discoveries that led to the proposal of new viral families, such as Polymycoviridae, Alternaviridae and, in the context of the present review, Exartaviridae. Finally, the major issue of phenotypic effects of mycoviral infection on the host is addressed, including aflatoxin production in $A$. flavus, together with growth and virulence in $A$. fumigatus. Although the molecular mechanisms behind these phenomena are yet to be elucidated, recent studies suggest that by implication, RNA silencing may be involved.

Keywords: mycovirus, Aspergillus, population study, mycovirus classification, mycovirus transmission, hypervirulence, hypovirulence

\section{INTRODUCTION}

The diversity of known fungal viruses, or mycoviruses, has increased rapidly over the last few years and this trend is expected to continue, especially with the development and the widespread use of state-of-the-art RNA deep sequencing techniques. Currently, the International Committee for the Taxonomy of Viruses (ICTV) officially recognizes 17 taxa, 16 families and one genus that does not belong to a family; these accommodate (exclusively or not) mycoviruses with double-stranded (ds) RNA linear genomes, positive-sense, single-stranded (ss) RNA linear genomes including reverse transcribing RNA linear genomes, negative-sense ssRNA linear genomes or ssDNA circular genomes (Figure 1). No mycoviruses with dsDNA genomes have been fully characterized at the molecular level yet. Since the majority of mycoviruses do not have an extracellular phase in their replication cycle, often their genomes are unencapsidated or non-conventionally encapsidated and true virions, if present, are proteinaceous in nature. To date, enveloped virions have not been discovered. 


\section{dsRNA mycoviruses}

Totiviridae

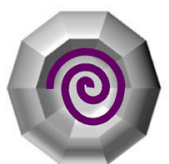

isometric virions $\mathbf{4 0} \mathbf{n m}$ 1 segment $4.6-7 \mathrm{kbp}$

\section{Partitiviridae}

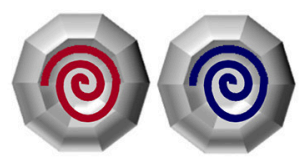

isometric virions $30-42 \mathrm{~nm}$ 2 segments 1.4-2.4 kbp
Chrysoviridae
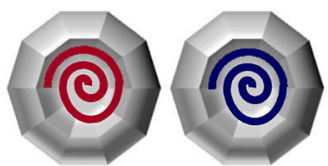

isometric virions $40 \mathrm{~nm}$ 4 segments 2.4-3.6 kbp

\section{Quadriviridae}
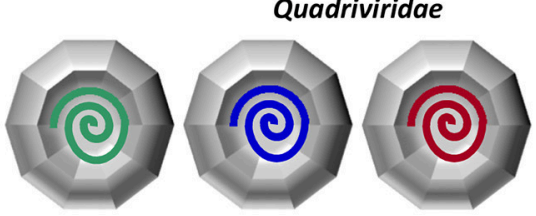

isometric virions $48 \mathrm{~nm}$

4 segments 3.7-4.9 kbp
Botybirnavirus

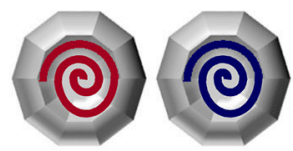

isometric virions $35 \mathrm{~nm}$ 2 segments 5.9-6.2 kbp

Megabirnaviridae

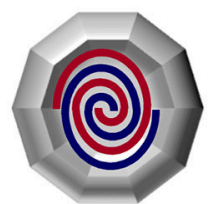

isometric virions $50 \mathrm{~nm}$ 2 segments 7-9 kbp

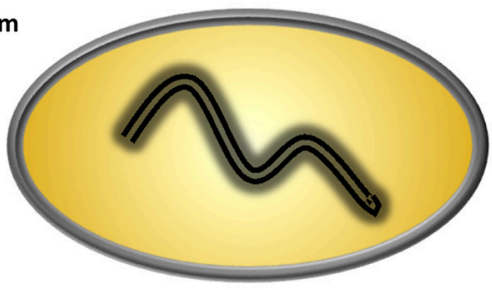

lipid vescicles 1 segment 14-17.6 kbp

\section{(+) SsRNA mycoviruses}

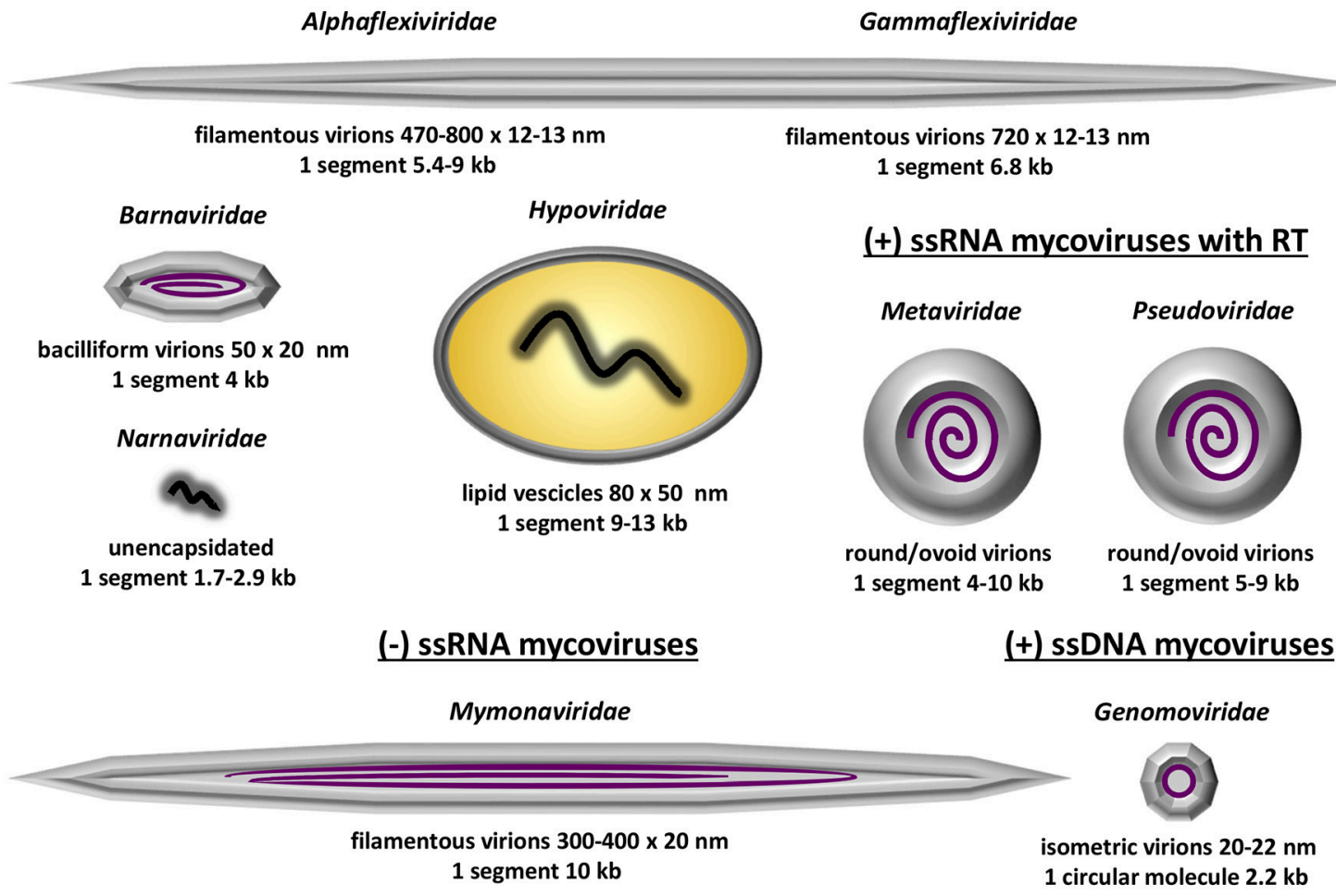

FIGURE 1 | Schematic representation of the 13 established mycovirus families; 6 families containing dsRNA as genetic material, 5 families containing ssRNA as genetic material and 2 families containing ssRNA that is reverse transcribed. Depicted is the virion structure and number of genomic segments for each family. The information was derived from Virus Taxonomy: The Classification and Nomenclature of Viruses-The Online (10th) Report of the ICTV; https://talk.ictvonline.org/ictvreports/ictv_online_report/. 
The dsRNA mycoviruses are the most commonly found and are classified in seven families and one genus: (1) Totiviridae (totus = "whole" in Latin) have non-segmented genomes and icosahedral virions. Genera Totivirus and Victorivirus infect exclusively fungi, while other totiviruses infect protozoa. (2) Partitiviridae (partitus = "divided" in Latin) have two genomic segments, each one encapsidated separately in distinct icosahedral virions. Genus Gammapartitivirus infect exclusively fungi, genera Alphapartitivirus and Betapartitivirus, infect both plants and fungi, genus Deltapartitivirus exclusively infect plants and genus Cryspovirus infect protozoa. (3) Megabirnaviridae $($ megas $=$ "large" in Greek $+b i=$ "two" in Greek + RNA) have two genomic segments, encapsidated together in icosahedral virions. The sole genus in the family exclusively infects fungi. (4) Botybirnavirus (boty from Botrytis, the host fungus, $+b i=$ "two" in Greek + RNA) have two genomic segments encapsidated in spherical virions and exclusively infect fungi. (5) Chrysoviridae were first isolated from the ascomycete Penicillium chrysogenum and have four genomic segments. Similarly to partitiviruses, each one of the four segments is encapsidated separately in distinct icosahedral virions. Chrysoviruses infect exclusively fungi. (6) Quadriviridae (quattuor = "four" in Latin) have four genomic segments encapsidated in spherical virions and the sole genus exclusively infect fungi. (7) Reoviridae (respiratory enteric orphan viruses) have 10-12 segments, which, unlike partitiviruses and chrysoviruses, are encapsidated together in a large icosahedral virion. Out of the fifteen genera comprising the family, only genus Mycoreovirus infect fungi while the rest infect plants, algae, invertebrates and vertebrates including humans. (8) Endornaviridae (endos = "within" in Greek + RNA) possess nonsegmented genomes that are non-conventionally encapsidated and accommodated in host-derived lipid vesicles. Both genera Alphaendoviridae and Betaendoviridae infect fungi, while the former also infect plants and oomycetes.

The positive-sense ssRNA mycoviruses all accommodate non-segmented genomes and are classified into five families: (1) Alphaflexiviridae genomes are encapsidated in filamentous virions and only genera Botrexvirus and Sclerodarnavirus infect fungi while the other five infect plants. (2) Gammaflexiviridae are very similar to Alphaflexiviridae, with genomes encapsidated in filamentous virions. The sole genus, Mycoflexivirus, exclusively infect fungi. Notably, Alphaflexiviridae and Gammaflexiviridae are the only mycoviral families that belong to an order, Tymovirales. (3) Barnaviridae (bacilliform $\underline{R N A}$ ) have genomes encapsidated in bacilliform virions and exclusively infect fungi. (4) Hypoviridae (hypo = "reduced" in Greek) have nonconventionally encapsidated genomes and are accommodated in host-derived lipid pleomorphic vesicles. Hypoviruses exclusively infect fungi and often cause significant hypovirulence. (5) Narnaviridae (naked $\underline{R N A}$ ) are the smallest, simplest known viruses with unencapsidated genomes. Genus Mitovirus are the most commonly found exclusively infect fungi and are localized in mitochondria. Genus Narnavirus infect fungi, oomycetes and protista and are localized in the cytoplasm. The positive-sense ssRNA mycoviruses that encode a reverse transcriptase enzyme and are incorporated into the hosts' genome are classified in two families, both forming round to ovoid virus like particles (VLPs):
(1) In the Metaviridae genus Metavirus include species that infect fungi. (2) In Pseudoviridae genera Hemivirus and Pseudovirus include species that also infect fungi.

The family Mymonaviridae (myco + mona) and its sole genus Sclerotimonavirus (sclerot $\bar{i}$ from Sclerotinia, the host fungus, + mona) in the order Mononegavirales accommodate the only negative-sense ssRNA virus known to infect fungi, a linear molecule encapsidated in filamentous virions. Although the family exclusively infect fungi, the order Mononegavirales accommodate important human pathogenic viruses, including the family Pneumoviridae and the genera Ebolavirus, Morbillivirus (cause of measles) and Lyssavirus (cause of rabies).

Finally, the family Genomoviridae, genus Gemycircularvirus $($ gemini-like + myco $+\underline{\text { circular }})$ accommodate the only ssDNA virus known to infect fungi, a circular molecule encapsidated in isometric virions. Other members of the genus and the family were derived from environmental, plant-associated or animalassociated material ${ }^{1}$.

Aspergillus is a genus of filamentous fungi belonging to the class Eurotiomycetes, phylum Ascomycota and comprising $>250$ species. Aspergillus is one of the most well studied fungi with a worldwide distribution occupying numerous niches, while individual Aspergillus species are medically, ecologically and economically important (Rokas, 2013). Investigations on Aspergillus mycoviruses over the past 50 years have been extensive, leading to numerous ground-breaking discoveries and enhancing our understanding of viral diversity and the significance of virus infection/symbiosis in fungi.

In the light of the above, this review aims to present the current knowledge on Aspergillus mycoviruses, from their initial identification, population and transmission studies to their phenotypes, their molecular characterisation and the discovery of novel virus families.

\section{INCIDENCE OF MYCOVIRUSES IN ASPERGILLI}

The presence of mycoviruses in Aspergillus species was initially reported in 1970, when Aspergillus foetidus strain IMI 41871 and $A$. niger strain IMI 146891 were found to harbor spherical VLPs containing dsRNA (Banks et al., 1970). Notably these $A$. foetidus dsRNA elements were fully sequenced and characterized only recently (see "The A. foetidus mycovirus complex" section below). Since the 1980's screening large panels of Aspergillus isolates revealed the presence of dsRNA elements in various species (Tables 1, 2). Traditionally screening is performed by extracting total nucleic acids from the isolates of interest and visualizing extra, non-chromosomal bands by agarose electrophoresis. The dsRNA nature of these elements is demonstrated following enzymatic treatment with DNase I (degrades dsDNA), S1 nuclease (degrades ss nucleic acids), and RNase A in high salt conditions (degrades ssRNA) and low salt

${ }^{1}$ All the information on virus families was derived from Virus Taxonomy: The Classification and Nomenclature of Viruses - The Online (10th) Report of the ICTV; https://talk.ictvonline.org/ictv-reports/ictv_online_report/. 
TABLE 1 | Incidence of dsRNA mycoviruses in Aspergilli.

\begin{tabular}{|c|c|c|c|c|}
\hline & Isolates screened & Isolates infected & Prevalence & References \\
\hline \multirow[t]{3}{*}{ Aspergillus section Nigri } & $>300$ & $35(12 \%)$ & Indonesia/Tunis/India & Varga et al., 1994 \\
\hline & 35 & $17(50 \%)$ & Indonesia/Netherlands & van Diepeningen et al., 1997 \\
\hline & 668 & $64(10 \%)$ & worldwide & van Diepeningen et al., 2006 \\
\hline Aspergillus section Circumdati & 27 & $4(15 \%)$ & Hungary/Zair & Varga et al., 1998 \\
\hline Aspergillus section Flavi & 92 & $10(11 \%)$ & U.S.A & Elias and Cotty, 1996 \\
\hline Aspergillus section Clavati & 8 & $2(25 \%)$ & Hungary/U.S.A & Varga et al., 2003 \\
\hline \multirow[t]{2}{*}{ Aspergillus section Fumigati } & 366 & $25(7 \%)$ & U.K. & Bhatti et al., 2012 \\
\hline & 86 & $16(19 \%)$ & Netherlands & Refos et al., 2013 \\
\hline
\end{tabular}

conditions (degrades ssRNA and dsRNA since the hydrogen bonds between the two RNA strands are more relaxed). The presence of VLPs associated with the dsRNA elements is shown by VLP purification and transmission electron microscopy (TEM).

\section{Aspergillus Section Nigri}

Black Aspergilli, or members of the Aspergillus section Nigri, are ubiquitous and include some of the most common Aspergillus species, such as $A$. niger. These fungi play an important role in agriculture and the food industry as plant infectious agents and food contaminants, together with medicine as a causative agent of aspergillosis. Black Aspergilli are also used in the fermentation industry to produce enzymes and chemicals (Abarca et al., 2004). Screening of $>300$, mostly Indonesian isolates revealed the presence of dsRNA elements, ranging in size from 0.5 to $5 \mathrm{kbp}$, in thirty five (ca. 12\%) black Aspergilli isolates, including eleven A. niger, five A. japonicus, three A. foetidus, three A. tubingensis, two A. heteromorphus, one A. carbonarius, and one A. awamori. The electrophoretic profiles of several isolates were identical or similar to that of $A$. foetidus strain IMI 41871, but homology was not examined. Isometric VLPs ca. 30-35 nm and occasionally 23$25 \mathrm{~nm}$ in size were observed in the Indonesian isolates (Varga et al., 1994). A subsequent investigation showed that almost half (17/35) black Aspergilli isolates, 11 A. niger, 3 A. tubingensis, 1 A. japonicas, and 1 A. carbonarius, harbor dsRNA elements. Similarly, all but one of the virus-infected isolates originated from Indonesia (van Diepeningen et al., 1997). Further and more comprehensive screening revealed that 64/668 (ca. 10\%) black Aspergilli isolates from all over the world were found to harbor up to eight dsRNA elements, ranging in size from 0.8 to $4.4 \mathrm{kbp}$ encapsidated in isometric particles $25-40 \mathrm{~nm}$ in diameter (van Diepeningen et al., 2006).

The presence of mycoviruses has also been reported in several other Aspergillus species, including members of the sections Circumdati, Flavi, Clavati and Fumigati.

\section{Aspergillus Section Circumdati}

These Aspergilli are often used for biochemical transformations; they produce proteolytic enzymes, metabolites, mycotoxins and promising anti-cancer compounds (Visagie et al., 2014). In $A$. ochraceus the presence of mycoviruses was initially reported by Kim and Bozarth (1985), who discovered isometric
VLPs $30-32 \mathrm{~nm}$ in diameter containing 9 segments of dsRNA. Subsequently, 4/27 (15\%) A. ochraceus isolates examined contained dsRNA elements ranging in size from 0.4 to $7 \mathrm{kbp}$ (Varga et al., 1998). The presence of dsRNAs and VLPs 36-40 nm in diameter was also noted in A. petrakii (Varga et al., 2001).

\section{Aspergillus Section Flavi}

Several species are used in food fermentation and biotechnological applications; importantly, A. flavus and other species in this section produce aflatoxins (Varga et al., 2011). A. flavus isolate NRRL 5565 contains isometric particles 27 and $30 \mathrm{~nm}$ in diameter (Wood et al., 1974; Gussack et al., 1977; Schmidt et al., 1986) and three dsRNA elements each ca. $3 \mathrm{kbp}$ in size (Schmidt et al., 1986). Isolate NRRL 5940 also harbors three dsRNAs, approximately 3.7, 3.4, and $2.9 \mathrm{kbp}$ in size and encapsidated in isometric particles $33 \mathrm{~nm}$ in diameter (Silva et al., 2001). Subsequently, 10/92 (11\%) isolates of the Aspergillus section Flavi, five A. flavus, three A. tamarii, one A. nomius and one $A$. parasiticus, were found to contain 1-9 dsRNA elements $0.4-10 \mathrm{kbp}$ in size (Elias and Cotty, 1996). The presence of dsRNAs and VLPs $36-40 \mathrm{~nm}$ in diameter was also noted in Aspergillus leporis (Varga et al., 2001).

\section{Aspergillus Section Clavati}

These Aspergilli are significant food contaminants, since they produce numerous mycotoxins including patulin (Varga et al., 2007). In total, $2 / 8$ (25\%) A. clavatus strains were found to harbor isometric particles $35-40 \mathrm{~nm}$ in diameter (Varga et al., 2003). The monopartite $6 \mathrm{kbp}$ dsRNA genome within the particles was considered an indication that these mycoviruses belong to the genus Totivirus, but this assumption has not been confirmed by sequence analysis.

\section{Aspergillus Section Fumigati}

A. fumigatus is one of the most important human fungal pathogens, which causes aspergillosis in immunocompromised patients (Brakhage and Langfelder, 2002). In total, 25/366 (333 clinical and 33 environmental; ca. 7\%) A. fumigatus isolates contained dsRNA elements (Bhatti et al., 2012). The electrophoretic pattern for seven of the isolates was characteristic for members of the family Partitiviridae (two dsRNAs, 1.5$2.0 \mathrm{kbp}$ in size) and identical to that of Aspergillus fumigatus partitivirus-1 (AfuPV-1; Bhatti et al., 2011a). Similarly, five 


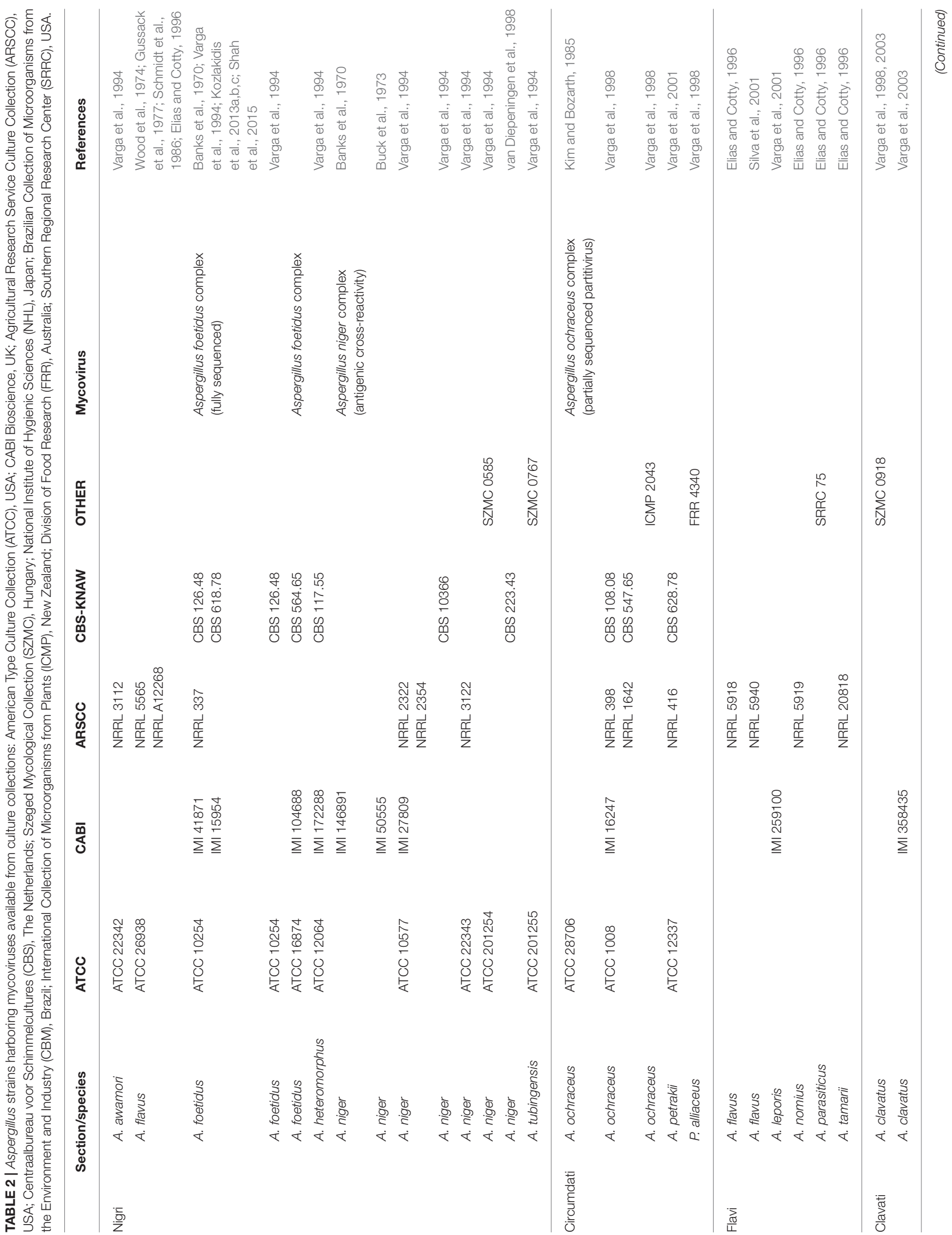




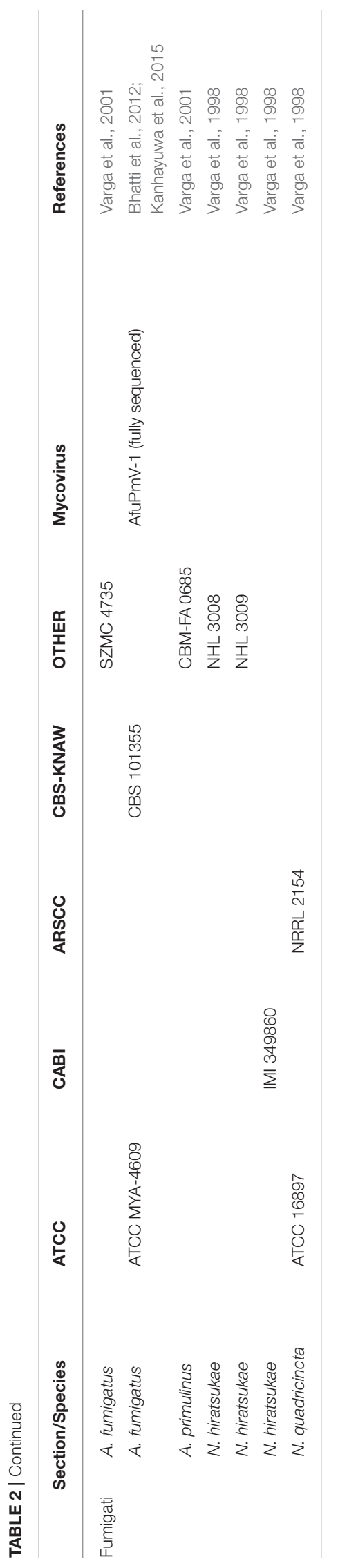

isolates contained four dsRNAs of a size and profile characteristic for members of the family Chrysoviridae and identical to that of Aspergillus fumigatus chrysovirus (AfuCV; Jamal et al., 2010). Finally, fourteen isolates contained four dsRNAs 1-2.5 kbp in size with a pattern identical to Aspergillus fumigatus tetramycovirus-1 (AfuTmV-1; Kanhayuwa et al., 2015) were found. Occasionally, some isolates appeared to be infected with more than one mycovirus (Bhatti et al., 2012). In another study, 16/86 (19\%) clinical A. fumigatus isolates were found to harbor three to five dsRNA elements, ranging in size from 0.87 to $3 \mathrm{kbp}$ (Refos et al., 2013).

\section{Sexual Aspergilli}

Although mycoviruses are common in asexual Aspergilli, they have been reported only rarely in sexual Aspergilli. More specifically, 1/5 Petromyces alliaceus (the teleomorph of A. alliaceus) strains examined was shown to harbor two dsRNA elements 3.5 and $0.6 \mathrm{kbp}$ in size where interestingly, the small element was localized in the mitochondrial fraction (Varga et al., 1998). Additionally, dsRNA elements and VLPs 36-40 nm in size were also observed in an A. primulinus isolate (Varga et al., 2001). In contrast, no dsRNA elements were discovered in a population study of 112 sexual Aspergillus nidulans isolates from Europe, Africa, America and Asia (Coenen et al., 1997). Finally, all three Neosartorya hiratsukae strains and $1 / 3$ N. quadricincta strains exhibited similar but not identical patterns of dsRNA elements ranging in size from 1.3 to $3.4 \mathrm{kbp}$ (Varga et al., 1998).

The presence and the quantity of dsRNA elements in Aspergilli have been reported to be dependent on the composition of the growth medium (Elias and Cotty, 1996). Since the dsRNA elements and the VLPs could not be detected in young mycelia ( $<48 \mathrm{~h}$ growth) and hyphal tips, it was suggested that at least some of these viruses multiply during the later phases of the fungal life cycle (Varga et al., 1994). In contrast, Silva et al. (2001) report that in A. flavus strain NRRL 5940 the number of viral particles increased during the exponential phase of growth and decreased during the later phases. No association between the presence of dsRNAs and the isolates' mitochondrial RFLP haplotype (van Diepeningen et al., 2006), specific conserved surface protein types or mating type MAT1-1 and MAT1-2 (Refos et al., 2013) have been observed.

\section{HORIZONTAL AND VERTICAL MYCOVIRUS TRANSMISSION}

An extracellular phase in their replication cycle has not been reported for the majority of known mycoviruses, which spread vertically via production of sexual and asexual spores and horizontally via hyphal anastomosis and heterokaryosis. This lack of extracellular transmission route is attributed to the physical barrier of the fungal cell wall that generally does not allow for the direct uptake of viruses. However, some exceptions have been noted in recent years; for instance, Sclerotinia sclerotiorum hypovirulence-associated DNA virus 1, a gemycircularvirus, has both an extracellular phase being infectious as viral particles (Yu 
et al., 2013) and a transmission vector, the mycophagous insect Lycoriella ingenua (Liu et al., 2016).

In Aspergilli, the transfer of dsRNA elements to asexual conidia is very efficient (Coenen et al., 1997; van Diepeningen et al., 1997; Varga et al., 1998); however this is not the case for sexual ascospores. Coenen et al. (1997) in an attempt to explain the paucity of dsRNA elements in Emericella nidulans, the teleomorph of $A$. nidulans, established that the elements are not transmitted via ascospores unless ascospores were produced by self-fertilization. This observation was subsequently confirmed for N. hiratsukae, a species phylogenetically close to Aspergillus (Varga et al., 1998).

Of particular interest is how the viruses are transmitted from one fungal strain to another and the role of heterokaryon incompatibility between fungal isolates as a barrier to mycovirus spread. Varga et al. (1994) demonstrated that viruses can be transferred between isogenic or vegetatively compatible A. niger strains using polyethylene-glycol protoplast fusion. Occasionally not all dsRNA elements of the donor were transferred successfully to the recipient. Similar experiments were performed by van Diepeningen et al. (1997, 1998), confirming the restrictions posed by vegetative incompatibility and applying mechanical disturbance of mycelia to allow virus transfer between incompatible strains via the released cytoplasm. Interestingly, vegetative compatibility appeared to be irrelevant to mycovirus spread between A. nidulans strains (van Diepeningen et al., 1998); however, as described for the plant pathogenic ascomycete Sclerotinia homoeocarpa, different levels of (in)compatibility between fungal strains have been recognized, with distinct effects on mycovirus transmission (Deng et al., 2002). Additionally, mycovirus transmission has been correlated with the number of different vegetative incompatibility genes between the strains tested in another plant pathogenic ascomycete, Cryphonectria parasitica (Liu and Milgroom, 1996). Different viruses can co-exist in the same host (van Diepeningen et al., 1998), but also limiting competition among the mycoviruses was reported since extant infected isolates could not accommodate additional dsRNA elements (van Diepeningen et al., 1997).

Regarding intraspecies transmission, it has been successfully demonstrated between $A$. niger (donor) and A. oryzae and $A$. ficuum (recipients) by Liang and Chen (1987), A. niger (donor) and $A$. tubingensis (recipient) by Varga et al. (1994) and van Diepeningen et al. (1998), A. niger (donor) and A. nidulans (recipient) by Coenen et al. (1997) and van Diepeningen et al. (1998), A. nidulans (donor) and A. niger (recipient) by van Diepeningen et al. (1998), supporting the notion that these viruses are not restricted in one species. Interestingly, they do not appear to be restricted in hyphomycetes either: Lhoas (1972) reported using a virus suspension from A. niger strain IMI 146891 to infect the unicellular ascomycete Saccharomyces cerevisiae, a process successful solely during the mating of the yeast cells. The virus particles produced in the yeast cells postinfection were localized in vacuoles as aggregates or in the cytoplasm and appeared identical to the original ones in the hyphae of the filamentous fungus, confirming the ability of the virus to replicate in its new host (Border, 1972).

\section{MOLECULAR CHARACTERISATION OF THE ASPERGILLUS MYCOVIRUSES}

Despite the extensive population studies described in the previous section only a small number of mycoviruses found in Aspergillus have had their genomes fully sequenced and annotated. This information is crucial for assessing the diversity of the virome in Aspergillus species, since the electrophoretic profiles of the dsRNA elements on agarose gels cannot be reliably used to infer evolutionary relationships with other viruses. To date, known members of the Aspergillus virome belong to well-established families such as Partitiviridae, Totiviridae and Chrysoviridae and provisionally designated families such as Polymycoviridae and Alternaviridae that accommodate novel viruses (Figure 2, Table 3).

\section{The Aspergillus foetidus Mycovirus Complex}

The dsRNA elements in A. foetidus strain IMI 41871 were among the first mycoviruses to be discovered (Banks et al., 1970) and constituted a model system in the 1970's for virus replication, transcription and biochemical studies (Ratti and Buck, 1975, 1978; Buck and Ratti, 1977), although the actual viruses were not sequenced and classified until recently.

Aspergillus foetidus strain IMI 41871 harbors seven dsRNA elements and two classes of VLPs. The VLPs were designated as fast (AfV-F) and slow (AfV-S) according to their electrophoretic mobility. Both AfV-F and AfV-S are isometric viruses, 33-37 nm in size (Ratti and Buck, 1972) and serologically unrelated to one another (Buck and Ratti, 1975). Each type is estimated to contain approximately 120 molecules of the respective coat protein (Buck and Ratti, 1975).

The AfV-F particles contain four dsRNAs, ca. 3.6, 2.8, 2.5, and $2.0 \mathrm{kbp}$ in size, respectively, each one encapsidated in separate virions. These four dsRNAs constitute the genome of a novel unclassified virus, provisionally designated as Aspergillus foetidus alternavirus (AfAV; Kozlakidis et al., 2013c). Each dsRNA has a single open reading frame (ORF), conserved $5^{\prime}$ - and $3^{\prime}$ terminal sequences that may be involved in replication and a $3^{\prime}$ poly (A) tail ranging in length from 17 to $62 \mathrm{nt}$. The largest dsRNA encodes the viral RNA dependent RNA polymerase (RdRP), $127 \mathrm{kDa}$ in size, and classified in one of the viral RdRP families (RdRP_4, pfam02123). The other three dsRNAs encode polypeptides of unknown function $87.5,79$, and $65 \mathrm{kDa}$ in size, respectively (Kozlakidis et al., 2013c). The $87.5 \mathrm{kDa}$ protein is probably the virus coat protein (CP) since its size corresponds to that of the major virion protein (Buck and Ratti, 1975).

The AfV-S particles contain three dsRNAs, ca. 5.2, 3.6, and $0.4 \mathrm{kbp}$ in size, respectively. The largest dsRNA is provisionally designated as Aspergillus foetidus victorivirus-1 (AfVV-1) and its genomic organization is typical of members of the genus Victorivirus, family Totiviridae, with two overlapping ORFs expressed via a coupled termination/reinitiation mechanism. The upstream ORF encodes the $\mathrm{CP}, 78 \mathrm{kDa}$ in size, while the downstream ORF encodes the viral RdRP, $92 \mathrm{kDa}$ in size (Kozlakidis et al., 2013a). The second dsRNA element is 
Aspergillus foetidus slow virus

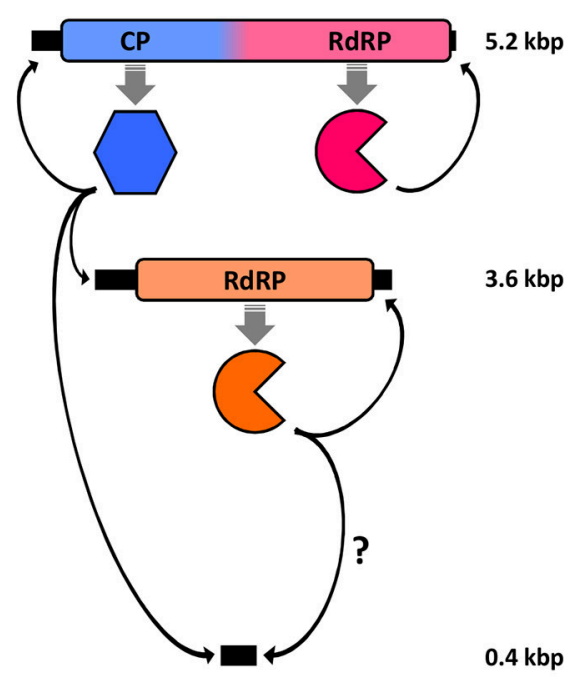

Aspergillus foetidus fast virus

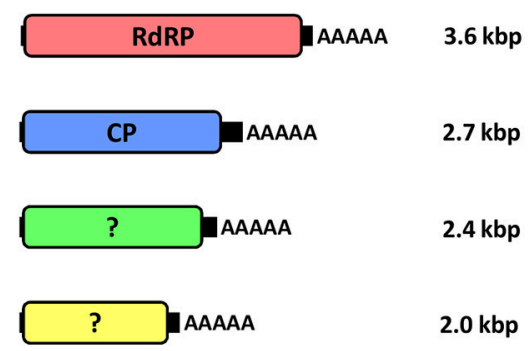

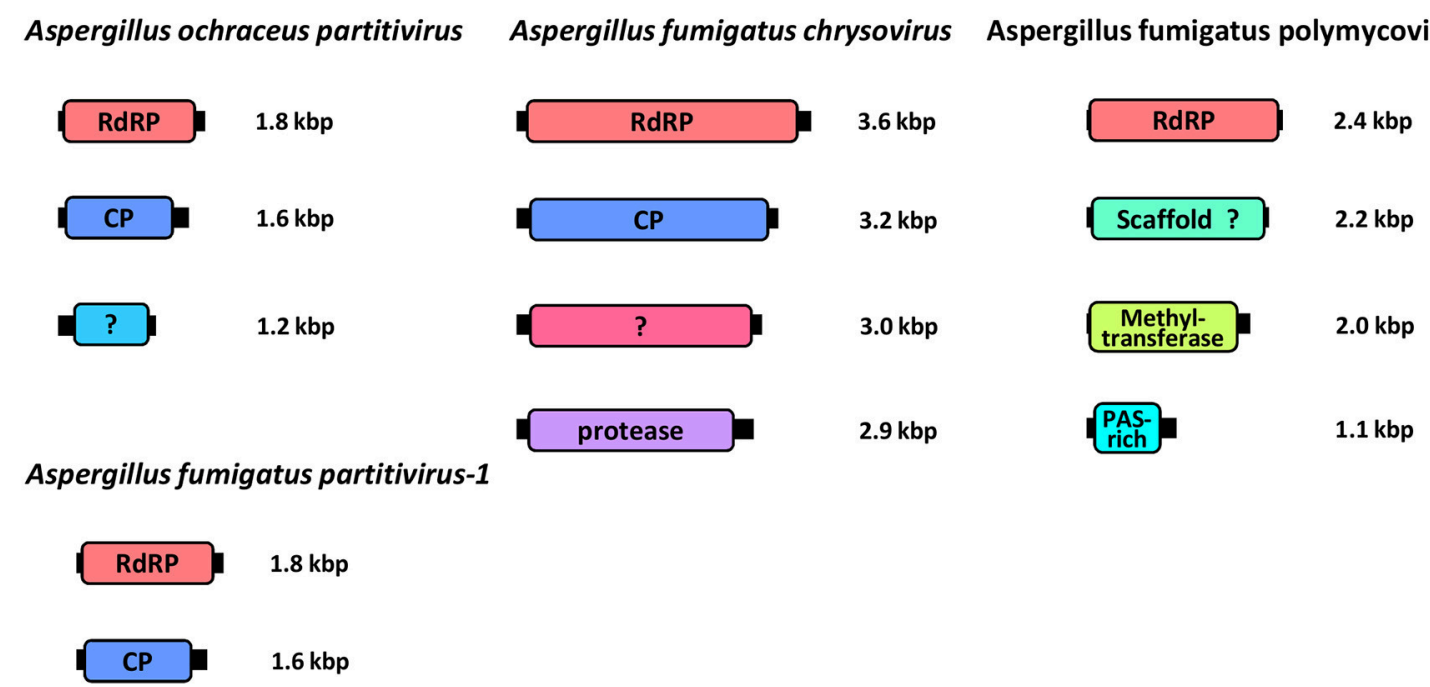

FIGURE 2 | Schematic representation of the genomic organization of all fully sequenced Aspergillus mycoviruses, including the $A$. foetidus mycovirus complex, the $A$. ochreacus and $A$. fumigatus partitiviruses and the $A$. fumigatus chrysovirus and polymycovirus. For each genomic segment, the ORF(s) (colored boxes) are flanked by $5^{\prime}$ - and $3^{\prime}$-UTRs (black boxes) and the function of the encoded protein(s) is indicated.

provisionally designated Aspergillus foetidus Exartavirus (AfExV; exarta- = "dependent" (for encapsidation) in Greek), contains an ORF encoding an RdRP, $100 \mathrm{kDa}$ in size, and an unusually long $5^{\prime}$-UTR (Kozlakidis et al., 2013b). The smallest dsRNA is a non-coding satellite RNA with a $5^{\prime}$-terminal sequence identical to that of AfExV (Shah et al., 2015) suggesting that both molecules are replicated by $A f E x V$ RdRP, while all three dsRNAs are encapsidated by the AfVV-1 CP.

The Aspergillus foetidus viruses were the first to be reported in Aspergillus sp. and appear to be rather common as identical or similar electrophoretic patterns have been reported for other isolates (Liang and Chen, 1987; Varga et al., 1994); however, without further molecular or biochemical evidence no definitive conclusions can be drawn. A similar virus complex has been described in A. niger isolates IMI 146891 (Banks et al., 1970) and IMI 50555 (Buck et al., 1973) and antigenic crossreactivity between the $A$. foetidus and $A$. niger viruses was also demonstrated (Buck et al., 1973). Interestingly, in A. niger IMI 50555 the second largest dsRNA that would correspond to the exartavirus, is encapsidated by both AnV-F and AnV-S particles, a phenomenon called genomic masking (Buck et al., 1973).

Individual viruses comprising the $A$. foetidus complex have been discovered in other fungal species as well. For instance the family Totiviridae is well established and contains the genus Victorivirus comprising fourteen official members plus the partially sequenced dsRNA Aspergillus 
TABLE 3 | Properties of known Aspergillus mycoviruses.

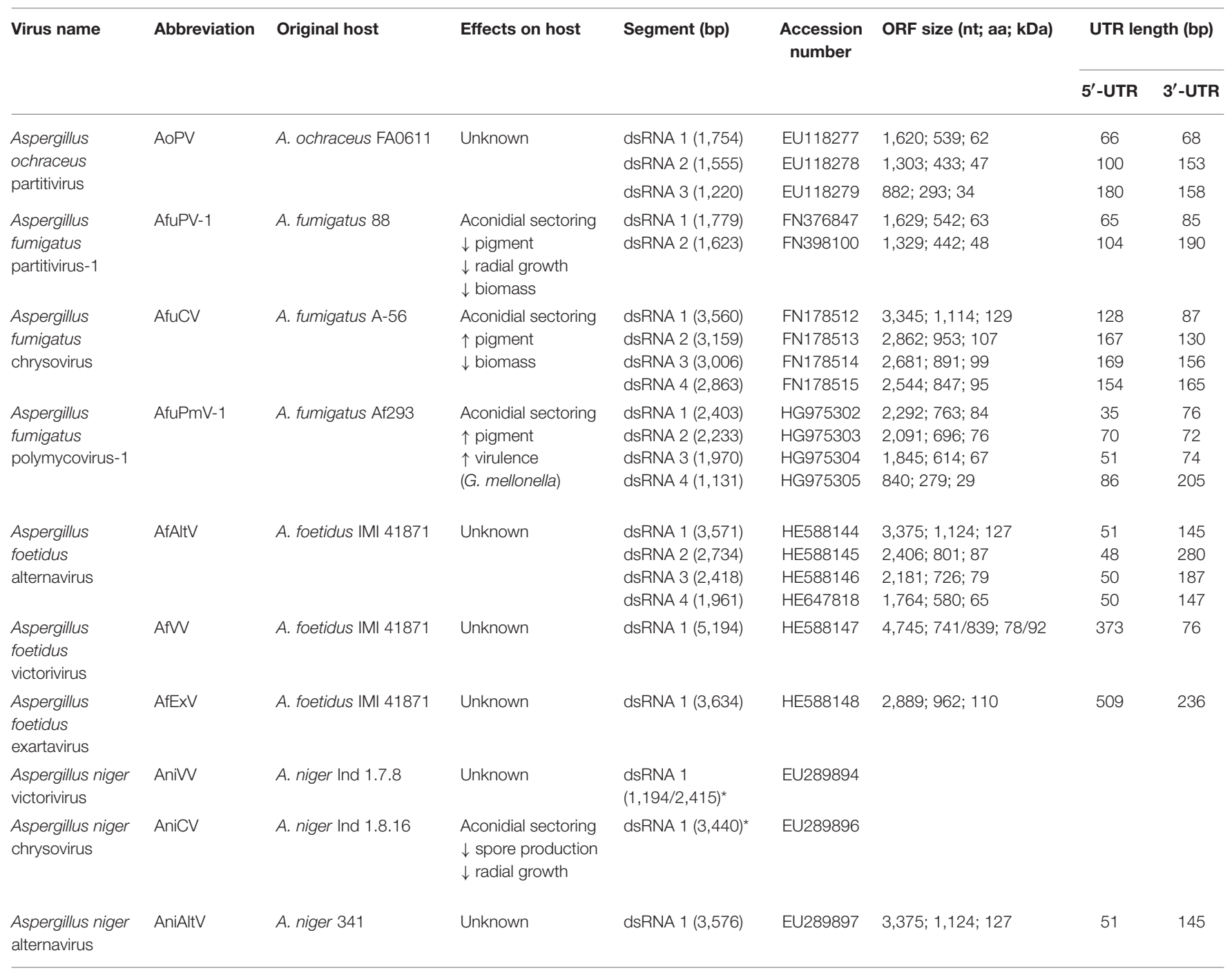

${ }^{\star}$ Partial sequence.

niger victorivirus-1 (AniVV-1) ca. $6 \mathrm{kbp}$ in size isolated from A. niger strain Ind 1.7.8 (Hammond et al., 2008). In this investigation two fragments 1.2 and $2.4 \mathrm{kbp}$ is size are significantly similar in sequence to the CP and RdRP genes of respectively Magnaporthe oryzae virus 3 and Penicillium aurantiogriseum totivirus 1, both established victoriviruses.

The proposed family Exartaviridae would include AfEx, Penicillium aurantiogriseum foetidus-like virus (Nerva et al., 2016), Rosellinia necatrix mycovirus 2-W1032/S6 (Zhang et al., 2016) and Fusarium poae mycovirus 2 (Osaki et al., 2016), isolated from ascomycetes, and Rhizoctonia solani mycovirus 1 (Bartholomäus et al., 2016), isolated from a basidiomycete (Figure 3). Interestingly, the exartaviruses have been argued to be positive-sense ssRNA viruses with a dsRNA replicative form (Zhang et al., 2016). The proposed family Alternaviridae includes Alternaria alternata alternavirus-1 (Aoki et al., 2009) as its prototype member, AfAV and Fusarium poae alternavirus
1 (Osaki et al., 2016), both isolated from ascomycetes. Additionally, another alternavirus from Aspergillus has been partially sequenced (Hammond et al., 2008); Aspergillus niger alternavirus (AniAltV), originally isolated from $A$. niger strain 341 , has four dsRNA segments, ranging in size from 1.5 to 3.6 $\mathrm{kbp}$, as its genome where the largest was sequenced and found encode an RdRP with 99\% similarity to AfAltV RdRP.

\section{Partitiviruses in Aspergillus}

Two members of the genus Gammapartitivirus, family Partitiviridae have been isolated from Aspergillus, Aspergillus ochraceus virus (AoV) from A. ochraceus strain FA0611 (Liu et al., 2008) and Aspergillus fumigatus partitivirus-1 (AfuPV-1) from A. fumigatus isolate 88; (Bhatti et al., 2011a). A strain of the former has been also found in A. ochraceus ATCC 28706 (Kim et al., 2006). Both viruses are typical members of their genus, with two dsRNA segments ca. 1.8 and $1.6 \mathrm{kbp}$ in length as their genome, each containing one ORF. The large dsRNA 
encodes the viral RdRP, 62 and $63 \mathrm{kDa}$ for AoV and AfuPV-1, respectively, while the small dsRNA encodes the $\mathrm{CP}, 47$ and 48 $\mathrm{kDa}$ for AoV and AfuPV-1, respectively. For both viruses the $5^{\prime}$ - and $3^{\prime}$-termini of untranslated regions (UTRs) flanking the ORF are highly conserved (Liu et al., 2008; Bhatti et al., 2011a). Interestingly, AoV has a third component, a dsRNA ca. $1.2 \mathrm{kbp}$ in length that encodes a $34 \mathrm{kDa}$ protein of unknown function. The UTRs of this dsRNA are very similar to those of the other two AoV components, suggesting that it is replicated by the RdRP (Liu et al., 2008). An additional dsRNA has also been reported for an AfuPV-1-like virus from the environmental A. fumigatus isolate Exeter 10.2, although it has not been sequenced (Bhatti, 2011; Figure 4A). Other gammapartitiviruses known to have a homologous third dsRNA are Gremmeniella abietina RNA virus MS1 (Botella et al., 2015), Gremmeniella abietina RNA virus MS2 (Tuomivirta and Hantula, 2005), and Ustilaginoidea virens partitivirus (Zhang et al., 2013), all of which infect ascomycetes.

\section{Chrysoviruses in Aspergillus}

A member of the family Chrysoviridae has been isolated from $A$. fumigatus isolate A-56 by Jamal et al. (2010) and designated as Aspergillus fumigatus chrysovirus (AfuCV). The AfuCV genome consists of four dsRNAs, ca. 3.6, 3.2, 3.0, and $2.8 \mathrm{kbp}$ in size, respectively, each one containing an ORF flanked by conserved $5^{\prime}$ - and $3^{\prime}$-UTRs. The two largest dsRNAs encode the viral RdRP and $\mathrm{CP}, 128$ and $107 \mathrm{kDa}$ in size, respectively. AfuCV dsRNA3 and dsRNA4 encode two proteins 99 and $95 \mathrm{kDa}$ homologous to those encoded by dsRNA4 and dsRNA3 of typical chrysoviruses, a phenomenon observed previously for Penicillium chrysogenum virus and Verticillium dahliae chrysovirus 1 . The former protein contains a "phytoreo S7 domain," shares significant sequence similarity with the N-terminus of the RdRP and is considered to interact with it and play a role in viral RNA binding and packaging (Ghabrial, 2008), while the latter is a putative protease. Additionally, another chrysovirus has been found in $A$. niger strain Ind 1.8.16. Aspergillus niger chrysovirus-1 (AniCV-1) and consists of four dsRNAs, ranging in size from 2 to $3.7 \mathrm{kbp}$, and one $3.4 \mathrm{kbp}$ fragment derived from the largest dsRNA has been sequenced and found to encode an RdRP (Hammond et al., 2008).

\section{A Polymycovirus in Aspergillus fumigatus} Aspergillus fumigatus polymycovirus-1 (AfuPmV-1) is the prototype member of the proposed family Polymycoviridae (Kotta-Loizou and Coutts, 2017), originally named Tetramycoviridae (Kanhayuwa et al., 2015), and was derived from A. fumigatus strain Af293. The AfuPmV-1 genome consists of four dsRNAs, similarly to the proposed family Alternaviridae and the established family Chrysoviridae described above; however they are smaller in size, ca. 2.4, 2.2, 2.0, and 1.1 $\mathrm{kbp}$. The largest dsRNA encodes the viral RdRP, which is unique in that although related to the positive-stranded RNA
A

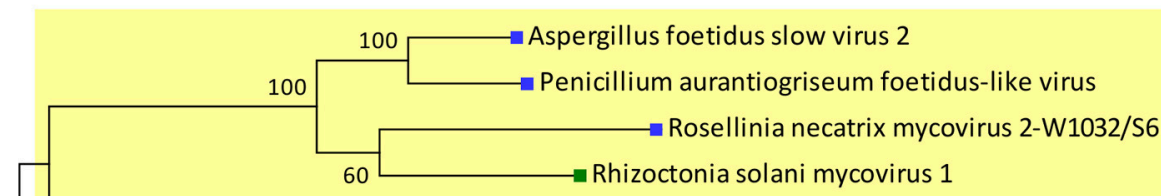

Fusarium poae mycovirus 2

$\rightarrow$ Bat calicivirus

B

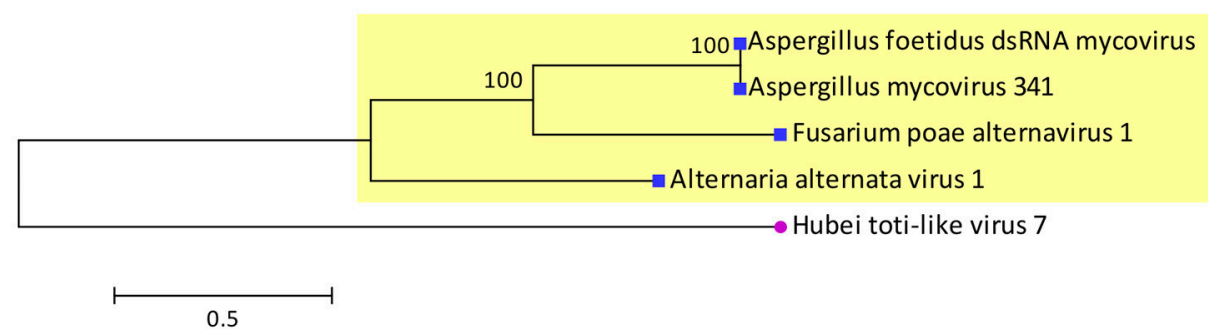

Exartaviridae

\section{Alternaviridae}

FIGURE 3 | Phylogenetic analysis of the proposed families (A) Exartaviridae and (B) Alternaviridae. Putative exartaviruses (RdRP_1, PF00680; Finn et al., 2014) include Aspergillus foetidus slow virus 2 (CCD33025), Penicillium aurantiogriseum foetidus-like virus (YP_009182156), Rosellinia necatrix mycovirus 2-W1032/S6 (BAT50982), Rhizoctonia solani mycovirus 1 (ANR02697), and Fusarium poae mycovirus 2 (YP_009272910). A bat calicivirus (AIF74264), the most closely related virus (PSI-BLAST; Altschul et al., 1997), was used as an outgroup. Putative alternaviruses (RdRP_4, PF02123; Finn et al., 2014) include Aspergillus foetidus dsRNA mycovirus (YP_007353985), Aspergillus mycovirus 341 (ABX79997), Fusarium poae alternavirus 1 (YP_009272952) and Alternaria alternata alternavirus-1 (YP_001976142). Hubei toti-like virus 7 (APG76025), the most closely related virus (PSI-BLAST; Altschul et al., 1997), was used as an outgroup. The RdRP sequences of the viruses were aligned with MUSCLE as implemented by MEGA 6 (Tamura et al., 2013) the alignment was improved manually and all positions with less than $30 \%$ site coverage were eliminated. Maximum likelihood phylogenetic trees were constructed with MEGA 6 using the WAG+G+I+F and LG+G substitution models for exartaviruses and alternaviruses respectively. At the end of the branches blue and green squares indicate that the virus infects ascomycetes and basidiomycetes respectively; red and purple circles indicate that the virus infects mammals and invertebrates respectively. 

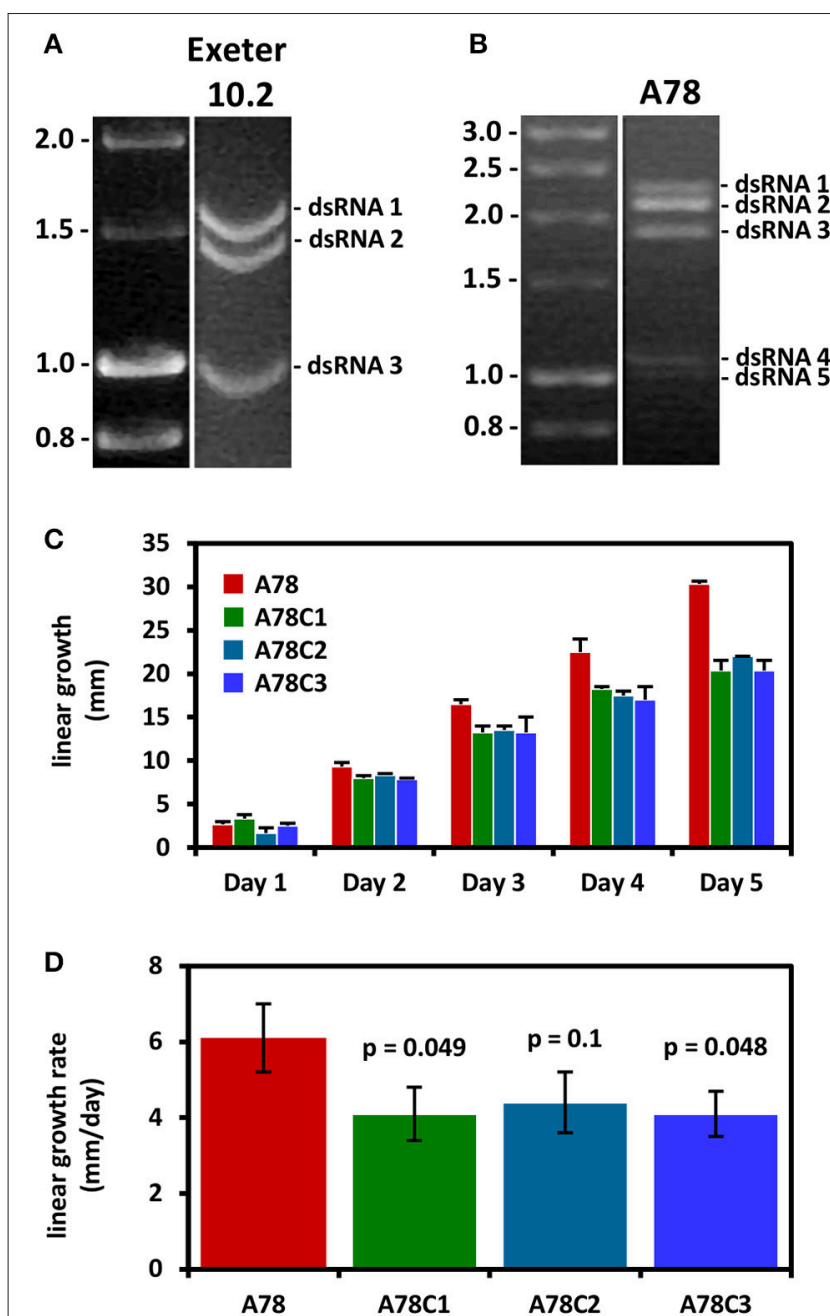

FIGURE 4 | (A) Agarose gel electrophoresis of dsRNA molecules from A. fumigatus strain Exeter 10.2 harboring a partitivirus. The dsRNAs 1 and 2 encode the RdRP and CP, respectively, while dsRNA 3 is an additional genomic component (adapted from Bhatti, 2011). (B) Agarose gel electrophoresis of dsRNA molecules from A. fumigatus strain A78, harboring a polymycovirus. A fifth dsRNA molecule is visible under the four dsRNAs constituting the viral genome (adapted from Readman, 2011). (C,D) Three virus-free isogenic colonies derived by curing the virus-infected $A$. fumigatus strain A78 exhibit reduced radial growth and radial growth rate on solid minimal medium. The $p$-values indicate the statistical significance of the difference in growth rate between the virus-infected and the three cured virus-free strains (Student's t-test; adapted from Readman, 2011).

picorna-superfamily has its catalytic GDD motif replaced with GDNQ, characteristic of negative-stranded RNA viruses. The second largest dsRNA encodes a protein of unknown function with a zinc finger-like motif and a putative ER retaining signal peptide. The third largest RNA encodes a methyl-transferase responsible for capping the positive RNA strands; notably this is the first report of a capping enzyme and the RdRP being on distinct dsRNA elements. Finally, the smallest dsRNA encodes a proline-alanine-serine (PAS) rich protein that is hypothesized to coat the viral genome, which is non-conventionally encapsidated. The virus was also shown to be infectious to A. fumigatus protoplasts as dsRNA (Kanhayuwa et al., 2015). Subsequently, members of the proposed family Polymycoviridae, such as those isolated from the insect pathogenic fungus Beauveria bassiana (Kotta-Loizou and Coutts, 2017) or the plant pathogenic fungus Botryosphaeria dothidea (Zhai et al., 2016), were discovered to have up to three additional small dsRNA elements encoding non-homologous proteins of unknown function. This is not the case for AfuPmV-1 but an uncharacterized virus with an identical electrophoretic profile isolated from A. fumigatus isolate A78 may harbor a fifth dsRNA segment (Readman, 2011; Figure 4B). Interestingly, a recently published related mycovirus from the plant pathogenic fungus Colletotrichum camelliae has four additional dsRNA segments, is also infectious as dsRNA and forms filamentous virions (Jia et al., 2017).

\section{EFFECTS OF MYCOVIRUSES ON THE FUNGAL HOST}

In order to reliably assess the effect of mycoviruses on their hosts it is necessary to produce virus-free and virus-infected isogenic lines, either by curing the virus-infected strain or by introducing the virus into a virus-free strain. Cycloheximide, a protein synthesis inhibitor, was successfully used to eradicate dsRNA mycoviruses from yeast (Fink and Styles, 1972) and subsequently from other fungi including Aspergilli (Elias and Cotty, 1996; Bhatti et al., 2011b). Cycloheximide-based infection curing is not always successful (Varga et al., 1998; van Diepeningen et al., 2006); Elias and Cotty (1996) reported a success rate of $40 \%$, while Bhatti et al. (2011b) managed to eradicate AfuCV but not AfuPV suggesting that both individual fungal strains and viruses might have different sensitivities to cycloheximide. Similarly, 5'fluorouracil has also been used in this context (Schmidt et al., 1986). Other methods that have been used for curing virusinfected fungi with various rates of success include single conidial isolation (Elias and Cotty, 1996; van Diepeningen et al., 2006), hyphal tipping (van Diepeningen et al., 2006) and selection for nitrogen-metabolism and other mutants (Varga et al., 1994; Elias and Cotty, 1996). Alternatively, isogenic lines can be produced by introducing the virus of interest into a virus-free strain, either via protoplast transfection or protoplast fusion. In examples where cycloheximide or other stress-inducing or mutagenic chemicals were originally used to eradicate the virus it is recommended the virus is reintroduced into the cured strain by protoplast transfection in order to ensure that any phenotypes observed are exclusively due to the presence of the virus.

The phenotypes commonly assessed by comparing virusfree and the virus-infected strains are pigmentation, growth, virulence and toxin production. Growth can be examined both in solid (radial growth) and in liquid (biomass production) medium, while growth media that differ in their nutritional values often serve to illustrate more clearly the effect of the virus on its host. To date, virulence in Aspergilli is determined using two model systems: the murine model, where the fungal burden in the lungs of immunosuppressed mice is measured by quantitative polymerase chain reaction, and larvae of the greater 
wax moth Galleria mellonella, where both survival rates and fungal burden are estimated (Özkan and Coutts, 2015).

In A. flavus strain NRRL 5565, the production of carcinogenic aflatoxins was shown to be repressed by the presence of a mycovirus (Schmidt et al., 1986). Similarly, in strain NRRL 5940, aflatoxin production occurred in parallel with decreased presence of viral particles (Silva et al., 2001). However Elias and Cotty (1996) reported that there was no correlation between the presence of dsRNA and the production of aflatoxin in other $A$. flavus isolates. In A. clavatus strains, the presence of mycoviruses was not associated with the production of the mutagenic patulin either (Varga et al., 2003). Since these mycotoxins, which are found on agricultural crops and may be consumed either directly or indirectly via meat and dairy products, represent a significant medical and economic issue especially in developing countries (Kumar et al., 2017), the idea of controlling their production using mycoviruses is very appealing. Unfortunately no further studies were performed to characterize the dsRNAs from the non-aflatoxin producing strain at the molecular level.

Virus-infected A. niger strain Ind 1.8.16 was observed to form aconidial sectors that demonstrated a reduced growth rate and increased dsRNA levels. A direct comparison of virus-infected and virus-free isogenic lines confirmed that the presence of dsRNA was associated with a statistically significant decrease in radial growth rate, spore production and competitive spore production that was more evident on minimal medium when compared to water agar, very minimal medium and complete medium (van Diepeningen et al., 2006).

The AfuCV-infected A. fumigatus isolate A-56 produces darker green pigmentation than the AfuCV-free isogenic strain and forms aconidial sectors. Additionally, its biomass in both minimal and Aspergillus complete liquid medium is significantly smaller (Bhatti et al., 2011b); however no statistically significant differences in virulence were observed between the AfuCV-free and the AfuCV-infected strain using the murine infection model (Bhatti et al., 2011b) and G. mellonella (Özkan and Coutts, 2015). Similarly, the AfuPV-1-transfected A. fumigatus isolate 237y demonstrated aconidial sectoring and lighter pigmentation when compared to the AfuPV-1-free isogenic strain, together with a significantly slower radial growth and biomass production in both minimal and Aspergillus complete medium (Bhatti et al., 2011b). Again no statistically significant differences in virulence were observed in the murine (Bhatti et al., 2011b) or G. mellonella (Özkan and Coutts, 2015) infection models. Comparison of the AfuPmV-1-infected A. fumigatus isolate 293 with a AfuPmV-1free isogenic strain revealed no statistically significant differences in radial growth rate or biomass production; however a statistically significant decrease in the survival rates of $G$. mellonella larvae infected with the AfuPmV-1-infected strain was observed. This was not accompanied with a concomitant increase in the fungal burden, suggesting that the hypervirulent effect is not due to fungal growth (Kanhayuwa et al., 2015). A. fumigatus isolate A78 harbors an uncharacterised virus with an electrophoretic profile identical to that of AfuPmV-1. Both virus-infected isolates show a minor increase in the intensity of pigmentation and form sectors (Kanhayuwa et al., 2015; Özkan and Coutts, 2015); however, in the case of isolate A78, the virusinfected strain demonstrated a statistically significant increase in radial growth rate that was more prominent in minimal than in Aspergillus complete medium. This was independently confirmed using three distinct cured isogenic virus-free colonies (Readman, 2011; Figure 4C). Additionally, a statistically significant decrease in the survival rates of $G$. mellonella larvae exposed to the virusinfected A78 isolate was noted, which was probably due to the faster growth of the strain as indicated by the fungal burden assay (Özkan and Coutts, 2015). Given the medical significance of $A$. fumigatus infections and the hypo- or hyper-virulent effects of the viruses on their host, it would be worthwhile to investigate further the presence of distinct mycoviruses in clinical samples in association with clinicopathological parameters and patient survival. Additionally, any potential links between mycoviruses and the worldwide problem of azole resistance (Perlin et al., 2017) should be elucidated. Aspergillosis is observed in patients with hematological malignancies or those who have undergone organ transplantation and, in the case of azole resistant strains, is associated with mortality rates up to $100 \%$ (Meis et al., 2016).

The molecular mechanisms underpinning these phenotypes in Aspergillus are still largely unexplored. However almost a decade ago, Hammond et al. (2008) indicated that at least some mycoviruses (as exemplified by AniCV) may suppress RNA silencing in A. nidulans and some others, such as the non-conventionally encapsidated AniAltV, may be silenced themselves as part of the anti-viral defense of their host strains. RNA silencing as an anti-mycoviral defense was initially studied in C. parasitica, where deletion of important genes encoding dicer-like or Argonaut-like proteins could lead to severe growth defects in the virus-infected but not the virus-free strains (Segers et al., 2007; Sun et al., 2009). Additionally, the papain-like protease encoded by Cryphonectria hypovirus 1 was identified as a RNA silencing suppressor both in fungi and plants (Segers et al., 2006). More recently, Özkan et al. (2017) demonstrated using high throughput small (s) RNA sequencing that nonconventionally encapsidated viruses, such as AfuPmV-1, and to a lesser extent encapsidated viruses, such as AfuPV-1 and AfuCV, are silenced in A. fumigatus. More specifically, $50 \%$ of total sRNAs where derived from AfuPmV-1, while the percentage was approximately half, 25 and $22 \%$ for AfuPV-1 and AfuCV, respectively. Additionally, sRNAs differentially expressed in virus-free and virus-infected samples were identified, suggesting a putative pathway for mediating observed mycovirus effects on fungal hosts (Özkan et al., 2017).

\section{CONCLUSION}

Mycoviruses in Aspergilli are more extensively studied than in any other fungal genus. The major established viral families, including Partitiviridae, Chrysoviridae, and Totiviridae, are well represented in Aspergillus species, while novel families such as Polymycoviridae, Alternaviridae and Exartaviridae have been proposed based on discoveries made in Aspergilli. Population studies and basic mycovirological 
research have provided valuable insights into the distribution and transmission of mycoviruses in medically, ecologically and economically important species. Finally, distinct phenotypes have been associated with the presence of mycoviruses although their molecular mechanisms are still unknown.

Future studies should focus on elucidating the molecular mechanisms of host-mycovirus interactions, especially the effects of the mycovirus infection on the fungal host. Offtarget RNA silencing is expected to play a role given the dsRNA nature of these viruses that act as both triggers and targets of the antiviral defense system. Additionally, host-virus protein-protein interactions via Eukaryotic Linear Motifs leading to disruption of host pathways may also be implicated in accordance with other host-pathogen systems. The mechanisms controlling mycovirus-induced phenotypic alterations, from reduced aflatoxin expression to hyper- or hypo-virulence are still

\section{REFERENCES}

Abarca, M. L., Accensi, F., Cano, J., and Cabañes, F. J. (2004). Taxonomy and significance of black Aspergilli. Antonie Van Leeuwenhoek 86, 33-49. doi: 10.1023/B:ANTO.0000024907.85688.05

Altschul, S. F., Madden, T. L., Schäffer, A. A., Zhang, J., Zhang, Z., Miller, W., et al. (1997). Gapped BLAST and PSI-BLAST: a new generation of protein database search programs. Nucleic Acids Res. 25, 3389-3402. doi: 10.1093/nar/25.17.3389

Aoki, N., Moriyama, H., Kodama, M., Arie, T., Teraoka, T., and Fukuhara, T. (2009). A novel mycovirus associated with four double-stranded RNAs affects host fungal growth in Alternaria alternata. Virus Res. 140, 179-187. doi: 10.1016/j.virusres.2008.12.003

Banks, G. T., Buck, K. W., Chain, E. B., Darbyshire, J. E., Himmelweit, F., Ratti, G., et al. (1970). Antiviral activity of double stranded RNA from a virus isolated from Aspergillus foetidus. Nature 227, 505-507.

Bartholomäus, A., Wibberg, D., Winkler, A., Pühler, A., Schlüter, A., and Varrelmann, M. (2016). Deep sequencing analysis reveals the mycoviral diversity of the virome of an avirulent isolate of Rhizoctonia solani AG-2-2 IV. PLoS ONE 11:e0165965. doi: 10.1371/journal.pone.0165965

Bhatti, M. F. (2011). Investigations on Aspergillus fumigatus Double-Stranded RNAs and Their Effects on the Fungus. $\mathrm{PhD}$ thesis, Imperial College London.

Bhatti, M. F., Bignell, E. M., and Coutts, R. H. A. (2011a). Complete nucleotide sequences of two dsRNAs associated with a new partitivirus infecting Aspergillus fumigatus. Arch. Virol. 156, 1677-1680. doi: 10.1007/s00705-011-1045-5

Bhatti, M. F., Jamal, A., Bignell, E. M., Petrou, M. A., and Coutts, R. H. A. (2012). Incidence of dsRNA mycoviruses in a collection of Aspergillus fumigatus isolates. Mycopathologia 174, 323-326. doi: 10.1007/s11046-012-9556-5

Bhatti, M. F., Jamal, A., Petrou, M. A., Cairns, T. C., Bignell, E. M., and Coutts, R. H. A. (2011b). The effects of dsRNA mycoviruses on growth and murine virulence of Aspergillus fumigatus. Fungal Genet. Biol. 48, 1071-1075. doi: $10.1016 /$ j.fgb.2011.07.008

Border, D. J. (1972). Electron microscopy of cells of Saccharomyces cerevisiae infected with double stranded RNA viruses from Aspergillus niger and Penicillium stoloniferum. Nat. N. Biol. 236, 87-88. doi: $10.1038 /$ newbio236087a0

Botella, L., Tuomivirta, T. T., Hantula, J., Diez, J. J., and Jankovsky, L. (2015). The European race of Gremmeniella abietina hosts a single species of Gammapartitivirus showing a global distribution and possible recombinant events in its history. Fungal Biol. 119, 125-135. doi: 10.1016/j.funbio.2014.12.001

Brakhage, A. A., and Langfelder, K. (2002). Menacing mold: the molecular biology of Aspergillus fumigatus. Annu. Rev. Microbiol. 56, 433-455. doi: 10.1146/annurev.micro.56.012302.160625 unclear but such knowledge would facilitate genetic engineering of known mycoviruses in order to induce desired phenomena. The discovery of non-conventionally encapsidated viruses which are infectious as dsRNA, such as polymycoviruses, will facilitate the process by providing us with tractable systems to study and engineer at will.

\section{AUTHOR CONTRIBUTIONS}

All authors listed have made a substantial, direct and intellectual contribution to the work, and approved it for publication.

\section{ACKNOWLEDGMENTS}

RHAC thanks The Leverhulme Trust for an Emeritus Fellowship award, and IKL acknowledges the support of the Erasmus European Union Programme.

Buck, K. W., and Ratti, G. (1975). Biophysical and biochemical properties of two viruses isolated from Aspergillus foetidus. J. Gen. Virol. 27, 211-224. doi: 10.1099/0022-1317-27-2-211

Buck, K. W., and Ratti, G. (1977). Molecular weight of double-stranded RNA: a re-examination of Aspergillus foetidus virus S RNA components. J. Gen. Virol. 37, 215-219. doi: 10.1099/0022-1317-37-1-215

Buck, K. W., Girvan, R. F., and Ratti, G. (1973). Two serologically distinct doublestranded ribonucleic acid viruses isolated from Aspergillus niger. Biochem. Soc. Trans. 1, 1138-1140.

Coenen, A., Kevei, F., and Hoekstra, R. F. (1997). Factors affecting the spread of double-stranded RNA viruses in Aspergillus nidulans. Genet. Res. 69, 1-10. doi: 10.1017/S001667239600256X

Deng, F., Melzer, M. S., and Boland, G. J. (2002). Vegetative compatibility and transmission of hypovirulence-associated dsRNA in Sclerotinia homoeocarpa. Can. J. Plant Pathol. 24, 481-488. doi: 10.1080/070606602095 07037

Elias, K. S., and Cotty, P. J. (1996). Incidence and stability of infection by doublestranded RNA genetic elements in Aspergillus section Flavi and effects on aflatoxigenicity. Can. J. Bot. 74, 716-725.

Fink, G. R., and Styles, C. A. (1972). Curing of a killer factor in Saccharomyces cerevisiae. Proc. Natl. Acad. Sci. U.S.A. 69, 2846-2849. doi: 10.1073/pnas.69.10.2846

Finn, R. D., Coggill, P., Eberhardt, R. Y., Eddy, S. R., Mistry, J., Mitchell, A. L., et al. (2014). Pfam: the protein families database. Nucleic Acids Res. 42, D222-D2230. doi: 10.1093/nar/gkt1223

Ghabrial, S. A. (2008). "Chrysoviruses”, in Encyclopaedia of Virology 3rd Edn., eds B. W. J. Mahy, and M. H. V. van Regenmortel (London: Elsevier/Academic Press), 503-513.

Gussack, G., Bennett, J. W., Cavalier, S., and Yatsu, L. (1977). Evidence for the parasexual cycle in a strain of Aspergillus flavus containing virus-like particles. Mycopathologia 61, 159-165. doi: 10.1007/BF00468010

Hammond, T. M., Andrewski, M. D., Roossinck, M. J., and Keller, N. P. (2008). Aspergillus mycoviruses are targets and suppressors of RNA silencing. Eukaryotic Cell 7, 350-357. doi: 10.1128/EC.00356-07

Jamal, A., Bignell, E. M., and Coutts, R. H. A. (2010). Complete nucleotide sequences of four dsRNAs associated with a new chrysovirus infecting Aspergillus fumigatus. Virus Res. 153, 64-70. doi: 10.1016/j.virusres.2010.07.008

Jia, H., Dong, K., Zhou, L., Wang, G., Hong, N., Jiang, D., et al. (2017). A dsRNA virus with filamentous viral particles. Nat. Commun. 8:168. doi: 10.1038/s41467-017-00237-9

Kanhayuwa, L., Kotta-Loizou, I., Özkan, S., Gunning, A. P., and Coutts, R.H.A. (2015). A novel mycovirus from Aspergillus fumigatus contains four unique dsRNAs as its genome and is infectious as dsRNA. Proc. Natl. Acad. Sci. U.S.A. 112, 9100-9105. doi: 10.1073/pnas.1419225112 
Kim, J. W., and Bozarth, R. F. (1985). Intergeneric occurrence of related fungal viruses: the Aspergillus ochraceus virus complex and its relationship to the Penicillium stoloniferum virus S. J. Gen. Virol. 66, 1991-2002. doi: 10.1099/0022-1317-66-9-1991

Kim, J. W., Choi, E. Y., and Kim, Y. T. (2006). Intergeneric relationship between the Aspergillus ochraceous virus $F$ and the Penicillium stoloniferum virus $S$. Virus Res. 120, 212-215. doi: 10.1016/j.virusres.2006.04.004

Kotta-Loizou, I., and Coutts, R. H. A. (2017). Studies on the virome of the entomopathogenic fungus Beauveria bassiana reveal novel dsRNA elements and mild hypervirulence. PLoS Pathog. 13:e1006183. doi: 10.1371/journal.ppat.1006183

Kozlakidis, Z., Herrero, N., and Coutts, R. H. A. (2013a). The complete nucleotide sequence of a totivirus from Aspergillus foetidus. Arch. Virol. 158, 263-266. doi: 10.1007/s00705-012-1368-x

Kozlakidis, Z., Herrero, N., Özkan, S., Bhatti, M. F., and Coutts, R.H.A. (2013b). A novel dsRNA element isolated from the Aspergillus foetidus mycovirus complex. Arch. Virol. 158, 2625-2628. doi: 10.1007/s00705-013-1779-3

Kozlakidis, Z., Herrero, N., Özkan, S., Kanhayuwa, L., and Jamal, A., Bhatti, M. F., et al. (2013c). Sequence determination of a quadripartite dsRNA virus isolated from Aspergillus foetidus. Arch. Virol. 158, 267-272. doi: $10.1007 / \mathrm{s} 00705-012-1362-3$

Kumar, P., Mahato, D. K., Kamle, M., Mohanta, T. K., and Kang, S. G. (2017). Aflatoxins: a global concern for food safety, human health and their management. Front. Microbiol. 7:2170. doi: 10.3389/fmicb.2016.02170

Lhoas, P. (1972). Mating pairs of Saccharomyces cerevisiae infected with double stranded RNA viruses from Aspergillus niger. Nat. N. Biol. 236, 86-87. doi: $10.1038 /$ newbio236086a0

Liang, P., and Chen, K. (1987). Virus transmission through interspecies protoplast fusion in Aspergillus. Trans. Br. Mycol. Soc. 89, 73-81.

Liu, S., Xie, J., Cheng, J., Li, B., Chen, T., Fu, Y., et al. (2016). Fungal DNA virus infects a mycophagous insect and utilizes it as a transmission vector. Proc. Natl. Acad. Sci. U.S.A. 113, 12803-12808. doi: 10.1073/pnas. 1608013113

Liu, W., Duns, G., and Chen, J. (2008). Genomic characterization of a novel partitivirus infecting Aspergillus ochraceus. Virus Genes 37, 322-327. doi: 10.1007/s11262-008-0265-6

Liu, Y. C., and Milgroom, M. G. (1996). Correlation between hypovirus transmission and the number of vegetative incompatibility (vic) genes different among isolates from a natural population of Cryphonectria parasitica. Phytopathology 86, 79-86. doi: 10.1094/Phyto-86-79

Meis, J. F., Chowdhary, A., Rhodes, J. L., Fisher, M. C., and Verweij, P. E. (2016). Clinical implications of globally emerging azole resistance in Aspergillus fumigatus. Philos. Trans. R. Soc. Lond. B. Biol. Sci. 371:pii: 20150460. doi: $10.1098 /$ rstb.2015.0460

Nerva, L., Ciuffo, M., Vallino, M., Margaria, P., Varese, G. C., Gnavi, G., et al. (2016). Multiple approaches for the detection and characterization of viral and plasmid symbionts from a collection of marine fungi. Virus Res. 219, 22-38. doi: $10.1016 /$ j.virusres.2015.10.028

Osaki, H., Sasaki, A., Nomiyama, K., and Tomioka, K. (2016). Multiple virus infection in a single strain of Fusarium poae shown by deep sequencing. Virus Genes 52, 835-847. doi: 10.1007/s11262-016-1379-x

Özkan, S., Coutts, R.H.A. (2015). Aspergillus fumigatus mycovirus causes mild hypervirulent effect on pathogenicity when tested on Galleria mellonella. Fungal Genet. Biol. 76, 20-26. doi: 10.1016/j.fgb.2015.01.003

Özkan, S., Mohorianu, I., Xu, P., Dalmay, T., and Coutts, R. H. A. (2017). Profile and functional analysis of small RNAs derived from Aspergillus fumigatus infected with double-stranded RNA mycoviruses. BMC Genomics 18:416. doi: 10.1186/s12864-017-3773-8

Perlin, D. S., Rautemaa-Richardson, R., and Alastruey-Izquierdo, A. (2017). The global problem of antifungal resistance: prevalence, mechanisms, and management. Lancet Infect. Dis. doi: 10.1016/S1473-3099(17)30316-X. [Epub ahead of print].

Ratti, G., and Buck, K. W. (1972). Virus particles in Aspergillus foetidus: a multicomponent system. J. Gen. Virol. 14, 165-175. doi: 10.1099/0022-1317-14-2-165

Ratti, G., and Buck, K. W. (1975). RNA polymerase activity in doublestranded ribonucleic acid virus particles from Aspergillus foetidus. Biochem. Biophys. Res. Commun. 66, 706-711. doi: 10.1016/0006-291X(75) 90567-7
Ratti, G., and Buck, K. W. (1978). Semi-conservative transcription in particles of a double-stranded RNA mycovirus. Nucleic Acids Res. 5, 3843-3854. doi: $10.1093 / \mathrm{nar} / 5.10 .3843$

Readman, J. (2011). Cloning and Sequencing dsRNA Elements from the Opportunistic Pathogen Aspergillus fumigatus. MRes Thesis, Imperial College London.

Refos, J. M., Vonk, A. G., Eadie, K., Lo-Ten-Foe, J. R., Verbrugh, H. A., van Diepeningen, A. D., et al. (2013). Double-stranded RNA mycovirus infection of Aspergillus fumigatus is not dependent on the genetic make-up of the host. PLoS ONE 8:e77381. doi: 10.1371/journal.pone.0077381

Rokas, A. (2013). Aspergillus. Curr. Biol. 23, R187-R188. doi: 10.1016/j.cub.2013.01.021

Schmidt, F. R., Lemke, P. A., and Esser, K. (1986). Viral influences on aflatoxin formation by Aspergillus flavus. Appl. Microbiol. Biotechnol. 24, 248-252.

Segers, G. C., van Wezel, R., Zhang, X., Hong, Y., and Nuss, D. L. (2006). Hypovirus papain-like protease p29 suppresses RNA silencing in the natural fungal host and in a heterologous plant system. Eukaryotic Cell 5, 896-904. doi: 10.1128/EC.00373-05

Segers, G. C., Zhang, X., Deng, F., Sun, Q., and Nuss, D. L. (2007). Evidence that RNA silencing functions as an antiviral defense mechanism in fungi. Proc. Natl. Acad. Sci. U.S.A. 104, 12902-12906. doi: 10.1073/pnas.0702500104

Shah, U. A., Kotta-Loizou, I., and Coutts, R. H. A. (2015). Sequence determination of a satellite RNA isolated from Aspergillus foetidus. Arch. Virol. 160, 883-885. doi: 10.1007/s00705-015-2338-x

Silva, V. N., Durigon, E. L., de Fátima Costa Pires, M., Lourenço, A., and de Faria, M. J., and Corrêa, B. (2001). Time course of virus-like particles (VLPs) doublestranded rna accumulation in toxigenic and non-toxigenic strains of Aspergillus flavus. Braz. J. Microbiol. 32, 56-60. doi: 10.1590/S1517-838220010001 00013

Sun, Q., Choi, G. H., and Nuss, D. L. (2009). A single Argonaute gene is required for induction of RNA silencing antiviral defense and promotes viral RNA recombination. Proc. Natl. Acad. Sci. U.S.A. 106, 17927-17932. doi: 10.1073/pnas.0907552106

Tamura, K., Stecher, G., Peterson, D., Filipski, A., and Kumar, S. (2013). MEGA6: molecular evolutionary genetics analysis version 6.0. Mol. Biol. Evol. 30, 2725-2729. doi: 10.1093/molbev/mst197

Tuomivirta, T. T., and Hantula, J. (2005). Three unrelated viruses occur in a single isolate of Gremmeniella abietina var. abietina type A. Virus Res. 110, 31-39. doi: 10.1016/j.virusres.2004.12.005

van Diepeningen, A. D., Debets, A. J., and Hoekstra, R. F. (1997). Heterokaryon incompatibility blocks virus transfer among natural isolates of black Aspergilli. Curr. Genet. 32, 209-217. doi: 10.1007/s002940050268

van Diepeningen, A. D., Debets, A. J., and Hoekstra, R. F. (1998). Intra- and interspecies virus transfer in Aspergilli via protoplast fusion. Fungal Genet. Biol. 25, 171-180. doi: 10.1006/fgbi.1998.1096

van Diepeningen, A. D., Debets, A. J., and Hoekstra, R. F. (2006). Dynamics of dsRNA mycoviruses in black Aspergillus populations. Fungal Genet. Biol. 43, 446-452. doi: 10.1016/j.fgb.2006.01.014

Varga, J., Due, M., Frisvad, J. C., and Samson, R. A. (2007). Taxonomic revision of Aspergillus section Clavati based on molecular, morphological and physiological data. Stud. Mycol. 59, 89-106. doi: 10.3114/sim.2007.59.11

Varga, J., Frisvad, J. C., and Samson, R. A. (2011). Two new aflatoxin producing species, and an overview of Aspergillus section Flavi. Stud. Mycol. 69, 57-80. doi: $10.3114 /$ sim.2011.69.05

Varga, J., Kevei, F., Vágvölgyi, C., Vriesema, A., and Croft, J. H. (1994). Doublestranded RNA mycoviruses in section Nigri of the Aspergillus genus. Can. J. Microbiol. 40, 325-329. doi: 10.1139/m94-054

Varga, J., Rigó, K., Molnár, J., Tóth, B., Szencz, S., Téren, J., et al. (2003). Mycotoxin production and evolutionary relationships among species of Aspergillus section Clavati. Antonie Van Leeuwenhoek 83, 191-200. doi: 10.1023/A:1023355707646

Varga, J., Rinyu, E., Kevei, E., Tóth, B., and Kozakiewicz, Z. (1998). Doublestranded RNA mycoviruses in species of Aspergillus sections Circumdati and Fumigati. Can. J. Microbiol. 44, 569-574. doi: 10.1139/cjm-44-6-569

Varga, J., Tóth, B., Szencz, S., Molnár, J., Fekete, C. S., and Szabó, L. (2001). Doublestranded RNA elements and virus-like particles in Aspergilli. Acta Biol. Hung. 52, 355-363. doi: 10.1556/ABiol.52.2001.2-3.20

Visagie, C. M., Varga, J., Houbraken, J., Meijer, M., Kocsubé, S., Yilmaz, N., et al. (2014). Ochratoxin production and taxonomy of the 
yellow Aspergilli (Aspergillus section Circumdati). Stud. Mycol. 78, 1-61.doi: 10.1016/j.simyco.2014.07.001

Wood, H. A., Bozarth, R. F., Adler, J., and Mackenzie, D. W. (1974). Proteinaceous virus-like particles from an isolate of Aspergillus flavus. J. Virol. 13, 532-534.

Yu, X., Li, B., Fu, Y., Xie, J., Cheng, J., Ghabrial, S. A., et al. (2013). Extracellular transmission of a DNA mycovirus and its use as a natural fungicide. Proc. Natl. Acad. Sci. U.S.A. 110, 1452-1457. doi: 10.1073/pnas.1213755110

Zhai, L., Xiang, J., Zhang, M., Fu, M., Yang, Z., Hong, N., et al. (2016). Characterization of a novel double-stranded RNA mycovirus conferring hypovirulence from the phytopathogenic fungus Botryosphaeria dothidea. Virology 493, 75-85. doi: 10.1016/j.virol.2016.03.012

Zhang, R., Hisano, S., Tani, A., Kondo, H., Kanematsu, S., and Suzuki, N. (2016). A capsidless ssRNA virus hosted by an unrelated dsRNA virus. Nat. Microbiol. 1:15001. doi: 10.1038/nmicrobiol.2015.1
Zhang, T., Jiang, Y., Huang, J., and Dong, W. (2013). Genomic organization of a novel partitivirus from the phytopathogenic fungus Ustilaginoidea virens. Arch. Virol. 158, 2415-2419. doi: 10.1007/s00705-013-1742-3

Conflict of Interest Statement: The authors declare that the research was conducted in the absence of any commercial or financial relationships that could be construed as a potential conflict of interest.

Copyright $\odot 2017$ Kotta-Loizou and Coutts. This is an open-access article distributed under the terms of the Creative Commons Attribution License (CC BY). The use, distribution or reproduction in other forums is permitted, provided the original author(s) or licensor are credited and that the original publication in this journal is cited, in accordance with accepted academic practice. No use, distribution or reproduction is permitted which does not comply with these terms. 